\title{
PAPERS
}

\section{A multicentre comparative trial of sodium valproate and carbamazepine in adult onset epilepsy}

\author{
A Richens, D L W Davidson, N E F Cartlidge, D J Easter on behalf of the Adult EPITEG \\ Collaborative Group
}

\begin{abstract}
The long term efficacy and safety of sodium valproate and carbamazepine in adult outpatients with newly diagnosed primary generalised or partial and secondarily generalised seizures were compared in a randomised, open, multicentre study at 22 neurology outpatient clinics. Patients were randomised to oral sodium valproate (Epilim EC enteric coated $200 \mathrm{mg}$ tablets twice daily, $\mathrm{n}=$ 149) or oral carbamazepine (100 mg twice daily increasing to $200 \mathrm{mg}$ twice daily in week $2, n=151$ ) and followed up for three years. If clinically necessary, dosages were regularly increased until seizures were controlled or toxicity developed. Sodium valproate and carbamazepine controlled both primary generalised and partial seizures equally effectively overall. Significantly more patients on sodium valproate than carbamazepine $(126 / 140(90 \%) v 105 / 141(75 \%), p=0.001)$ remained on randomised treatment for at least six months. Skin rashes occurred significantly more often in carbamazepine recipients than in sodium valproate recipients $(11.2 \% v 1.7 \%, \mathrm{p}<0.05)$ and carbamazepine was associated with a higher withdrawal rate because of adverse events $(15 \% v 5 \%$ on sodium valproate) in the first six months of treatment. There was no difference between the drugs in the rate of withdrawal because of poor seizure control at any stage, regardless of seizure type. At the end of the three year trial period, over $70 \%$ of the available patients were still on randomised treatment or had recently stopped treatment after achieving full seizure control. Sodium valproate and carbamazepine were both associated with a high degree of overall seizure control regardless of seizure type and both have good long term tolerability in adult patients with newly diagnosed epilepsy. Recommendations are made for a higher initial dosage regime for sodium valproate in partial seizures.
\end{abstract}

$(\Im$ Neurol Neurosurg Psychiatry 1994;57:682-687)
After a period when anticonvulsant polytherapy had been widely used in the treatment of epilepsy, it became apparent that a single drug was capable of controlling as many as $75 \%$ of adult outpatients with epilepsy. ${ }^{1}$ Several studies had indicated that seizure control was affected by seizure type. Carbamazepine was reported to be the treatment of choice for partial seizures and sodium valproate for absences. ${ }^{23}$ Turnbull et al compared phenytoin and sodium valproate monotherapy in 140 previously untreated adults for at least two years. ${ }^{4}$ Both drugs were found to be effective in the control of primary generalised and partial seizures, with a better response in patients with generalised attacks. Callaghan, et al compared carbamazepine, phenytoin, and sodium valproate in 181 previously untreated patients, and concluded that there was little difference in efficacy between the three drugs for either primary generalised or partial seizures. 5

We undertook this multicentre prospective trial to compare the long term efficacy and tolerability of sodium valproate and carbamazepine in untreated newly diagnosed adults with primary generalised or partial seizures with or without generalisation presenting to neurological clinics. The relation between dose and seizure control was also investigated.

Patients and methods

Three hundred patients with newly diagnosed idiopathic generalised tonic clonic seizures or partial seizures with or without generalisation were recruited for this three year study at 22 outpatient clinics in the United Kingdom.

To be eligible, patients (either sex) had to be over 16 years of age and to have had at least two generalised tonic-clonic or partial seizures with or without generalisation in the previous six months. Patients with both primary generalised tonic-clonic and other generalised seizures (for example, myoclonic or typical absence seizures) were included, but the efficacy analyses were based solely on the tonic-clonic seizure data.

Patients with an accompanying renal, hepatic, or CNS disorder, abnormal liver function tests, low platelet count, or other 
blood dyscrasia on initial assessment were excluded. Patients with absences or myoclonic jerks alone were excluded, as were female patients who were pregnant, lactating, or planning to become pregnant during the study. No concomitant anticonvulsants were permitted during the study; any patient who required additional antiepileptic medication during the study was withdrawn. All patients gave written or verbal informed consent before participating in the study, which was conducted in accordance with the Declaration of Helsinki (1964) as amended in Tokyo (1975) and which was approved by ethics committees local to each clinic.

On entry, patients were stratified by age, sex, seizure type, and centre and randomised by telephone to either sodium valproate $(n=$ $149)$ or carbamazepine $(n=151)$ by a computerised minimisation programme based in the Medical Department at Sanofi Winthrop Ltd.

The recommended initial dose for sodium valproate (Epilim enteric coated tablets) was $200 \mathrm{mg}$ orally twice daily; if clinically necessary, the dose could be increased by $200 \mathrm{mg}$ twice daily at weekly intervals until seizures were controlled or until toxic symptoms appeared, up to a maximum daily dose of $2400 \mathrm{mg}$. The recommended initial dosage of carbamazepine was $100 \mathrm{mg}$ orally twice daily, increasing in the second week to $200 \mathrm{mg}$ twice daily. Further increases of $200 \mathrm{mg} /$ day at fortnightly intervals were permitted as required to control seizures or until signs of toxicity appeared, up to a maximum of $1600 \mathrm{mg} /$ day. Patients in whom treatment was discontinued because of adverse events or whose seizure control was not satisfactory were regarded as treatment failures, were switched to the alternative drug, and were followed up for the rest of the three year study. A detailed analysis of their progress after failure on randomised treatment is outside the scope of this report.

\section{EFFICACY AND SAFETY ASSESSMENTS}

Patients were assessed at one month, then at three-monthly intervals for the first two years of the trial and at about six-monthly intervals thereafter (maximum duration of follow up seven years). An EEG taken at entry was used to aid classification of seizure type. ${ }^{6}$ Seizure control was assessed from a daily seizure diary completed by the patient. Serum drug concentrations were determined after three months' and 12 months' treatment, and at other times at the discretion of the investigator to monitor patient compliance. Routine haematology and liver function tests were carried out at entry, at three months, and then at the discretion of the investigator. To monitor drug safety, at each visit patients were asked whether they had been affected by treatment in any way, and any adverse events reported were recorded. Events were graded as severe if they warranted discontinuation of treatment. Treatment was continued if events were graded moderate or mild. All concomitant medication was recorded at each visit.
STATISTICAL ANALYSIS

Three hundred patients were recruited to this study, which was designed to detect an overall difference in seizure control of $15 \%-20 \%$ between treatments with $\alpha=0.05$ and $\beta=$ $0 \cdot 80$. All statistical tests were two tailed at the $5 \%$ significance level.

Seizure data were analysed on an intention to treat basis including all randomised patients with a diagnosis of epilepsy who received study treatment and who had adequate follow up data on treatment (at least one visit). Patients who failed to take their medication regularly or who showed poor compliance with the study medication were included in all efficacy analyses as if they had remained on treatment.

For the remission analysis, the seizure data recorded at each visit was used to determine the time to achieve six, 12 , and 24 months of freedom from seizures for each patient over the 36 month study period. Cumulative remission distributions were computed for each treatment group and expressed as unadjusted Kaplan-Meier plots, censored for severe adverse events, deaths, and dropouts.

Efficacy was also assessed by a retention analysis comparing the number of patients who remained in the study on randomised treatment. The Kaplan-Meier method was used to compute cumulative distributions for each treatment group, censoring for deaths and dropouts only.

The effects of all relevant prognostic factors (for example, treatment, seizure type, pretreatment seizure frequency, age, sex, etc) on the remission and retention analyses were analysed by Cox regression models. ${ }^{7}$

Safety was assessed in all patients who were on randomised treatment, or who had changed from one treatment to the other. Fisher's exact test was used to compare adverse event incidence between treatments for the randomised treatment period only.

The incidence of treatment failure due to severe adverse events or poor seizure control on each treatment was also compared with Fisher's exact test.

\section{Results}

\section{STUDY POPULATION}

A total of 140 of 149 patients randomised to sodium valproate and 141 of 151 patients randomised to carbamazepine could be evaluated on an intention to treat basis. The distribution of age, sex, weight, EEG, medical history, intellectual status, seizure type, and seizure frequency was similar in each treatment group, supporting the preliminary demographic findings of this study reported previously. ${ }^{8}$ The mean age of sodium valproate and carbamazepine recipients was 33 years and 34 years respectively. The seizure type at entry was classified on clinical grounds as "primary generalised" in 69 patients in each treatment group and as "partial with or without generalisation" in 71 sodium valproate recipients and in 72 carbamazepine recipients.

The EEGs done at or near entry were 
reported by the local hospital services as normal in $32 \%$ of patients, with $32 \%$ showing focal features and $11 \%$ generalised spike wave activity. Subsequently, an independent assessment of all available EEG records (by Dr C Binnie, Maudsley Hospital, London) indicated that a small proportion (about $20 \%$ ) of the patients with "primary generalised" seizures had focal features that could have indicated a partial origin to the seizures, but in the absence of clinical evidence of focal onset, the original investigator's assessment of seizure type at entry was used for all efficacy analyses. The reasons for exclusion of the 19 patients from the efficacy analyses were no data after randomisation (seven); incomplete data on treatment (seven); violation of entry criteria (not treated) (two); not epilepsy (two); and died in train incident (presumed suicide) (one).

\section{TREATMENT FAILURES}

Adverse events led to withdrawal of treatment in $7 / 140(5 \%)$ sodium valproate recipients and in 22/141 (15\%) carbamazepine recipients in the first six months of treatment. Poor seizure control resulted in treatment withdrawal in $4 / 140(3 \%)$ of sodium valproate recipients and $3 / 141(2 \%)$ of carbamazepine recipients over this period; all these patients had frequent partial seizures at entry. Significantly more sodium valproate recipients $(90 \%)$ remained in the study at six months compared with carbamazepine recipients $(75 \%, p=0.001)$.

Over the period from six months to three years, treatment failed because of poor seizure control in $15 / 126(12 \%)$ of sodium valproate recipients and in 12/105 (12\%) of carbamazepine recipients, and because of severe adverse events in $5 \%$ of sodium valproate recipients and $3 \%$ of carbamazepine recipients, respectively. Poor seizure control combined with adverse events at low drug doses was reported for two (2\%) additional patients on sodium valproate.

\section{EFFICACY ANALYSIS}

About half of the patients who were able to tolerate the trial drugs remained completely free of seizures for the first six months on treatment (fig 1); most of the remainder achieved at least six months' remission before

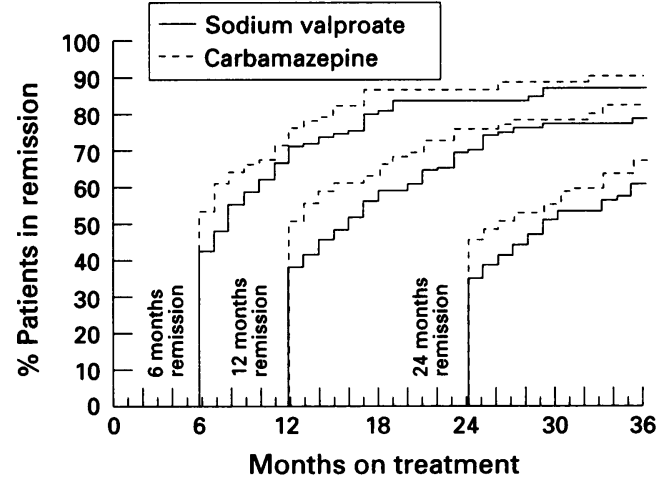

Figure 1 Remission analysis: percentage of all patients free of seizures for 6, 12, and 24 months.

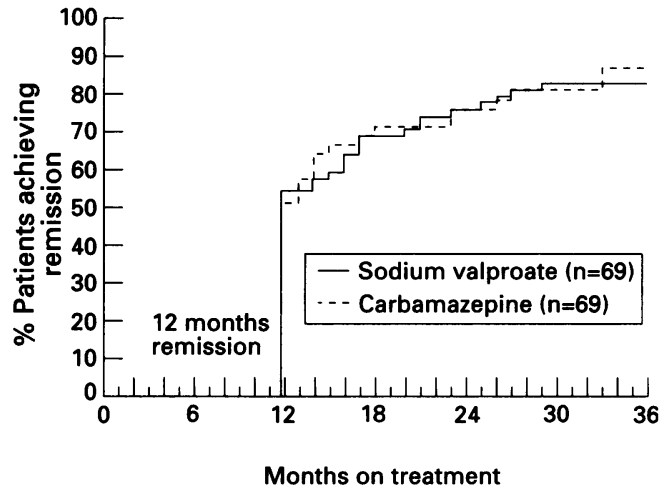

Figure 2 Remission analysis: percentage of patients free of seizures for 12 months: patients with primary generalised seizures.

the end of the three year trial period. By the end of three years, $80 \%$ of the patients available for assessment had achieved 12 months' remission and $60 \%$ had achieved 24 months' remission. A similar proportion of patients in each treatment group had achieved six, 12, and 24 months' remission by three years.

In patients with primary generalised seizures who tolerated the trial drugs, there was no difference between valproate and carbamazepine in the 12 month remission rates (fig 2). In partial seizures, similar overall 12 month remission rates were achieved for each treatment (sodium valproate $72 \%$, carbamazepine $76 \%$ ) by the end of the three year trial period (fig 3 ).

The full Cox model analysis, incorporating all the prognostic covariates showed that the differences in remission rates between the drugs were minimal, with a marginal and statistically insignificant advantage for carbamazepine compared with sodium valproate in the six month (risk ratio (RR) $0.92,95 \%$ confidence interval $(95 \% \mathrm{CI}) 0.69-1 \cdot 24), 12$ month $(R R=0.89,95 \% \mathrm{CI} 0.65-1 \cdot 21)$ and 24 month ( $R R=0.83$, 95\% CI $0.59-1 \cdot 19$ ) remission data. In strong contrast to this, the number of seizures during the six months before treatment had a significant effect on six month remission $(R R=0.52,95 \% \quad C I$ $0.28-0.97$ ) and a similar effect on 12 month $(R R=0.52,95 \% \quad C I \quad 0.27-1 \cdot 01)$ and 24 month remission $(R R=0.49$, 95\% CI $0 \cdot 23-1 \cdot 06$ ), indicating a considerably higher risk of failure on either drug for patients with four or more seizures before entry.

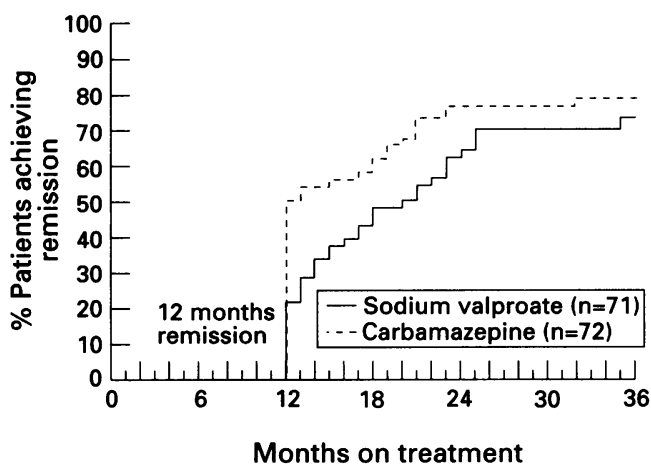

Figure 3 Remission analysis: percentage of patients free of seizures for 12 months: patients with partial seizures with or without generalisation. 
In these primary analyses, patients who were intolerant of the trial drugs were censored from the analysis from the time of withdrawal. Such patients could, however, be regarded clinically as treatment failures. In a subsidiary analysis retaining these patients as treatment failures, essentially similar results were obtained to those found in the primary analysis: Overall, patients on sodium valproate achieved higher six month $(79 \% v$ $72 \%, \mathrm{RR}=1 \cdot 21,95 \%$ CI $0.91-1.61)$ and 12 month $(69 \% v 64 \%, \mathrm{RR}=1 \cdot 14,95 \% \mathrm{CI}$ 0.84-1.55) remission rates but similar 24 month remission rates $(52 \% v 51 \%, R R=$ $1.01,95 \%$ CI $0.71-1.43$ ) to patients on carbamazepine: Patients with primary generalised seizures achieved higher 12 month remission rates on sodium valproate $(76 \%)$ than on carbamazepine $(62 \% \mathrm{RR}=1.41$, 95\% CI $0 \cdot 91-2 \cdot 18)$, whereas patients with partial seizures had similar overall remission rates $(62 \% \quad v 66 \%, \mathrm{RR}=0.84,95 \%$ CI $0.54-1 \cdot 30$ ) on either drug. None of these differences was statistically significant by Cox model analysis.

Cox model analysis of the proportion of patients remaining on treatment (retention analysis) showed that patients with primary generalised seizures who received sodium valproate were significantly more likely to remain on randomised treatment than were carbamazepine recipients $(R R=0.34, \quad C I$ $0 \cdot 16-0 \cdot 72$ ), with a non-significant advantage for carbamazepine in partial seizures $(R R=$ $1 \cdot 60,95 \%$ CI $0 \cdot 84-3 \cdot 05)$. There was a significant benefit $(R R=0.41,95 \%$ CI $0 \cdot 17-0.96)$ for sodium valproate over carbamazepine in younger patients (under 25 years old) and in patients who had had less than four seizures in the six months before the study $(R R=0.35$, 95\% CI 0.16-0.80).

Using the methodology given by Kay, ${ }^{9}$ a competing risks analysis was done by separating the Cox model into two componentsadverse events and poor seizure control (the combined category was ignored because of small numbers). For poor seizure control there was no difference between treatments $(\mathrm{RR}=1 \cdot 09,95 \%$ CI $0 \cdot 56-2 \cdot 11)$. For adverse events however, there was a significant treatment effect in favour of valproate $(R R=0.48$, 95\% CI 0.25-0.93).

\section{MEAN DRUG DOSE LEVELS}

Patients on sodium valproate reached a mean dose of $924 \mathrm{mg} /$ day at month 24, those with partial seizures requiring higher doses (mean $1066 \mathrm{mg} /$ day) than those with primary generalised seizures (mean $821 \mathrm{mg} /$ day). Patients on carbamazepine reached a mean dose of $516 \mathrm{mg} /$ day at two years and there was no difference between patients with primary generalised seizures (mean $520 \mathrm{mg} /$ day) and those with partial seizures (mean $511 \mathrm{mg} /$ day).

\section{ADVERSE EVENT ANALYSIS}

The adverse event analysis (which includes patients changed from one drug to the other) is based on reports from 174 patients treated with sodium valproate (165 for more than three months) and from 178 patients treated with carbamazepine (147 for more than three months). An interim report on part of these data has been presented previously. ${ }^{10}$

The overall prevalence of adverse events was similar in the two groups, 86 of 174 $(49 \cdot 4 \%)$ sodium valproate recipients and 87 of $178(48.9 \%)$ carbamazepine recipients reporting events at some time in the three year trial period.

The table shows the adverse events that were reported by four or more patients. Rashes occurred significantly more often on carbamazepine $(11.2 \%$ of patients) than on sodium valproate $(1.7 \%, \mathrm{p}<0.05)$ and for carbamazepine, were predominantly reported within the first three months of treatment. Other drug related adverse events with carbamazepine seemed to be dizziness $(6.7 \% v$ $2.9 \%)$, headaches $(6 \cdot 1 \% \vee 3.4 \%)$, and ataxia $(2 \cdot 2 \% v 0 \%)$ (all non-significant).

Significantly more patients on sodium valproate reported weight gain $(12 \cdot 1 \% v 1 \cdot 1 \%$, p $<0.05$ ), usually after at least three months on treatment. Other drug related adverse events with valproate seemed to be tremor $(5.2 \% v$ $1.7 \%)$, alopecia $(2.9 \% v 0.6 \%)$, and appetite increase $(2 \cdot 3 \% v 0 \%)$ (all non-significant).

Treatment was withdrawn because of adverse events in $9.3 \%$ of patients on valproate (no predominant event) and $17 \cdot 7 \%$ of patients on carbamazepine (predominantly rashes with or without other events). In the first six months on treatment, rash alone or in combination accounted for $13(59 \%)$ of the $22(15 \cdot 6 \%)$ withdrawals on carbamazepine. There was no clear pattern of adverse events for the seven patients (5\%) withdrawn from valproate treatment.

In the subsequent 30 months on treatment, six patients $(4.3 \%)$ on valproate and three patients $(2 \cdot 2 \%)$ on carbamazepine were withdrawn after adverse events. Late events on valproate were (a) rash and oedema, (b) alopecia, (c) thrombocytopenia, (d) tremor,

Table Adverse events reported by four or more patients at any time during treatment

\begin{tabular}{lcc}
\hline & \multicolumn{2}{l}{$\begin{array}{l}\text { Number of patients reporting } \\
\text { adverse event }\end{array}$} \\
\cline { 2 - 3 } Adverse event & $\begin{array}{l}\text { Sodium } \\
\text { valproate }\end{array}$ & Carbamazepine \\
\hline Fatigue & 15 & 17 \\
Somnolence & 12 & 20 \\
Nausea/vomiting/dyspepsia & 13 & 15 \\
Weight increase & $21^{\star \star}$ & 2 \\
Rash & 3 & $18^{\star \star}$ \\
Headache & 6 & 11 \\
Dizziness & 5 & $12^{\star}$ \\
Tremor & 9 & 3 \\
Amnesia & 6 & 3 \\
Depression & 4 & 4 \\
Pregnancy & 1 & 6 \\
Alopecia & 5 & 1 \\
Abnormal hepatic function & 2 & 3 \\
Ataxia & 0 & 4 \\
Appetite increase & 4 & 0 \\
Aphasia & 1 & 3 \\
Other events & 43 & 41 \\
Total no adverse events & 151 & 163 \\
\hline
\end{tabular}

${ }^{\star} p=0.05 ;{ }^{\star \star} p=0.01$ between treatments, Fisher's exact test. Data from 174 patients on valproate $(165$ for at least three months) and 178 patients on carbamazepine (147 for at least months) and 178 
extrapyramidal symptoms, raised liver enzymes, and heart failure, (e) somnolence and fatigue, and $(f)$ fatigue. Late events with carbamazepine were $(a)$ menstrual disorder and fatigue, $(b)$ psoriatric rash, and $(c)$ rash (unspecified).

The number of pregnancies on treatment reported during the study was higher among carbamazepine recipients $(3 \cdot 4 \%)$ than among sodium valproate recipients $(0 \cdot 6 \%)$. Including those patients who had stopped their treatment before or shortly after becoming pregnant, eight patients randomised to sodium valproate and 13 to carbamazepine became pregnant during the three year trial period. One patient on valproate elected for termination of pregnancy (no abnormalities reported) and one had a seizure during delivery. There were two adverse events reported for patients randomised to carbamazepine: one case of foetal malformation (pyloric stenosis) and one of premature birth. All other patients had normal pregnancies and deliveries.

Five deaths were reported during the trial, none of which was considered by the investigator to be drug related. Two deaths were in the valproate group (man aged 58 from cardiovascular disease and a man aged 31 in a train incident (presumed suicide) shortly after randomisation (no data on treatment)). Two deaths were in patients on carbamazepine (a woman aged 66 from a heart attack and a man aged 58 from cancer of the bronchus). The fifth patient (a woman aged 34) died of an astrocytoma nine months after withdrawal from the trial (on carbamazepine).

No clinically significant changes in haematology or biochemistry were noted on either treatment. White cell counts seemed to be depressed (by $8 \%$ ) on carbamazepine treatment, but the wide scatter in pretreatment levels limited any detailed interpretation of these data. Mean platelet counts were unchanged during treatment with either valproate or carbamazepine. One patient with an abnormally low platelet count (below $150 \times 10^{9}$ ) before treatment, received several months of treatment with valproate at doses up to $2000 \mathrm{mg} /$ day before falling platelet counts prompted drug withdrawal (with full recovery).

Alanine aminotransferase (ALT, SGPT) and aspartate aminotransferase (AST, SGOT) activities showed minor and insignificant changes during treatment with either drug. Alkaline phosphatase activity was extremely variable, particularly in younger patients with residual maturational effects, but absolute change, percentage change, transition, and frequency analyses indicated that carbamazepine treatment was associated with increased alkaline phosphatase (mean change $0-3$ months $+15 \%$ on carbamazepine $v+1 \%$ on valproate).

\section{Discussion}

Sodium valproate and carbamazepine are well established as safe and effective drugs for the treatment of all types of epilepsy (except that carbamazepine is not recommended in certain types of generalised seizures ${ }^{11}$ ). Until recently there has been a widely held view, however, (mainly from anecdotal evidence) that valproate was best for primary -generalised seizures, and carbamazepine for partial seizures. Our main objective in this trial was to compare directly these two drugs when used in the routine treatment of newly diagnosed adult onset epilepsy, both generalised and partial, in busy hospital neurology clinics.

The analysis of treatment failures showed that in the first six months of treatment 22 out of 141 patients on carbamazepine treatment $(15.6 \%)$ were withdrawn because of severe adverse events (mainly rashes in the first few weeks of treatment). Thereafter, there was little difference in failure rates between the treatments, whether for adverse events, poor seizure control, or both. At the end of the three year trial period, over $70 \%$ of the available patients were still on randomised treatment or had recently stopped treatment after achieving full seizure control.

The remission analyses showed that both sodium valproate and carbamazepine achieved a high degree of seizure control, $80 \%$ of patients available for assessment having had at least 12 months' freedom from seizures, and $60 \%$ at least two years' freedom over the three year trial period.

Although there were no differences in overall control, patients with partial seizures on sodium valproate seemed to take longer to achieve remission. This was most likely due to the low initial regime for sodium valproate and the protocol recommendation that the dose be increased only if needed to control seizures. Patients randomised to sodium valproate started at $200 \mathrm{mg}$ twice daily (current data sheet recommendation $600 \mathrm{mg} /$ day). Analysis of mean daily doses for valproate indicated a target dose of $821 \mathrm{mg} /$ day for primary generalised seizures, but a higher dose $(1066 \mathrm{mg} /$ day) to achieve similar control with partial seizures. For carbamazepine, there was little difference in mean daily dose for primary generalised seizures $(520 \mathrm{mg} /$ day $)$ and for partial seizures $(511 \mathrm{mg} /$ day).

These results indicate the need for a higher starting dose for sodium valproate in partial seizures than that used in this study. The present data sheet recommendation is 600 $\mathrm{mg} /$ day in divided doses rising as necessary to 1000-2000 mg/day. The results presented here suggest an increase to $800-1000 \mathrm{mg} /$ day soon after the start of treatment for patients with partial seizures regardless of response to ensure full early seizure control for both primary generalised and partial seizures in adults.

The results presented on efficacy are similar to those reported by Reynolds and his colleagues $^{12}$ who compared phenobarbitone, phenytoin, carbamazepine, and sodium valproate in adults with newly diagnosed epilepsy. In their study, all four drugs achieved similarly high degrees of seizure control, and there were no significant differences in efficacy between the drugs regardless of seizure type. 
Comparable efficacy of sodium valproate and carbamazepine in both primary generalised and partial seizures has also been shown in paediatric patients. Reynolds and colleagues $^{13}$ compared phenytoin, carbamazepine, and sodium valproate in children with newly diagnosed epilepsy and showed that all drugs achieved similarly high degrees of seizure control both overall and by seizure type and that there were no significant differences in efficacy between the drugs regardless of seizure type. In a paediatric version of the present trial, Verity et $a l^{4}$ showed that valproate and carbamazepine were equally effective in achieving high levels of seizure control in both primary generalised and partial seizures. In that trial, patients on valproate seemed to achieve better long term remission than those on carbamazepine, regardless of seizure type, although the differences were not statistically significant.

Our results differ from those of Mattson et $a l^{15}$ who reported that whereas there was no difference with secondary generalised tonicclonic seizures, carbamazepine provided significantly better seizure control than sodium valproate in adult patients with predominantly complex partial seizures. Their study was, however, carried out in a different and restricted patient group that was not representative of the range of patients with epilepsy presenting to neurology clinics; the patients were predominantly male war veterans, $31 \%$ of whom had trauma related epilepsy, with a mean age of 47 years. The study also had a considerably higher dropout rate $(27 \%$ in the first 12 months $v 5 \%$ in the present study). Furthermore, whereas over $98 \%$ of our patients had newly diagnosed epilepsy (none on treatment), in the study by Mattson et al, ${ }^{15}$ $50 \%$ of the patients had already been on medication and $26 \%$ were still on medication shortly before entry.

The adverse event profiles for sodium valproate and carbamazepine reported here are similar to those reported by these investigators and others, ${ }^{16}$ although the actual incidence of specific adverse effects seems to be very dependent on the treatment policy used (for example, minimal effective dose (as used here) $v$ target serum drug concentrations).

Treatment was withdrawn because of adverse effects in $18 \%$ of the patients on carbamazepine (50\% due to early rashes) and $9 \%$ of the patients on sodium valproate (no dominant event). There was a considerably higher number of pregnancies in patients on carbamazepine, but the data are not available to establish whether all of these were unintended or due to reduced efficacy of contraceptive medication after hepatic enzyme induction.

In conclusion, we have shown in this trial in adult patients with newly diagnosed seizures, that sodium valproate and carbamazepine were of similar overall efficacy whether for primary generalised seizures or partial seizures. Apart from a high incidence of severe rashes in the first few weeks of treatment with carbamazepine, both drugs showed equivalent long term tolerability. The results presented here and elsewhere would support an initial dosage regime for sodium valproate of $600 \mathrm{mg} /$ day for all seizure types, increasing to 800 or $1000 \mathrm{mg} /$ day within the first month in patients with partial seizures to ensure that full seizure control is achieved as soon as possible after start of treatment.

We gratefully acknowledge the advice, support, and statistical expertise provided by Dr A L Johnson (MRC Biostatistics Unit, Cambridge) and Mr M James (Sanof Winthrop Ltd) in the data analysis and to Mr P Simmonds (Sanofi Winthrop Ltd) for the data presentation. Our thanks go also to Dr C Binnie of the Maudlsey Hospital, London for his independent assessment of the pretreatment EEG records.

Other members of the Adult EPITEG Collaborative Group, without whose participation and enthusiasm the trial could not have been undertaken, were $\mathrm{Dr} D$ Bates, Royal Victoria Infirmary, Newcastle upon Tyne; Dr HG Boddie, North Infirmary, Newcastle upon Tyne; Dr HG Boddie, North Staffordshire Royal Infirmary, Stoke on Trent; Dr CRA Clarke, St Bartholomew's Hospital, London; Dr P Cleland Sunderland District General Hospital, Sunderland; Dr RN Corston, New Cross Hospital, Wolverhampton; Dr RE Cull,
Royal Infirmary, Edinburgh; Dr WJK Cumming, Withington Royal Infirmary, Edinburgh; Dr WJK Cumming, Withington Hospital, Manchester; Dr CJK Ellis, Poole General Hospital,
Poole; Dr AK Gupta, Dudley Road Hospital, Birmingham; Professor MJG Harrison, Middlesex Hospital, London; Dr G Harwood, St Bartholomew's Hospital, Rochester, Kent and Greenwich District Hospital, London; Dr D Jefferson, Derbyshire Royal Infirmary, Derby; Professor C Kennard, Charing Cross Hospital, London; Dr LA Loizou, Pinderfields General Hospital, Wakefield; Dr PAH Millac, Leicester Royal Infirmary, Leicester; Dr P Newman, Middlesbrough Genera Hospital, Middlesbrough; Dr VH Patterson, Claremont Street Hospital for Nervous Diseases, Belfast, and Dr DI Shepherd, North Manchester General Hospital, Manchester.

1 Reynolds EH, Shorvon SD. Monotherapy or polytherapy for epilepsy? Epilepsia 1981;22:1-10.

2 Jeavons PM, Covanis A, Gupta AK. Monotherapy with sodium valproate. In: Cranger $\mathrm{R}$, Angeleri F, Penry JK, ed. Advances in Epileptology. New York: Raven Press, 1980:415-8. (Epilepsy International Symposium No XII.)

3 Jeavons PM. Choice of drug therapy in epilepsy. Practitioner 1977;219:542-56.

4 Turnbull DM, Howel D, Rawlins MD, Chadwick DW. Which drug for the adult epileptic patient: phenytoin or valproate? BMF 1985;290:815-9.

5 Callaghan N, Kenny RA, O'Neill B, Crowley M, Goggin T. A prospective study between carbamazepine, phenytoin and sodium valproate as monotherapy in previously untreated and recently diagnosed patients with epilepsy. f Neurol Neurosurg Psychiatry 1985;48:639-44.

6 Commission on Classification and Terminology of the International League against Epilepsy. Proposal for revised clinical and electroencephalographic classification of epileptic seizures. Epilepsia 1981;22:489-501.

7 Cox DR. Regression models and life tables. $\mathcal{F} R$ Stat Soc Series $B$ 1972;34:187-220.

8 Cartlidge NEF. The adult EPITEG trial: a comparative multicentre clinical trial of sodium valproate and carbamazepine in adult onset epilepsy. Part 1: description of trial and demographic data. In: Chadwick D, ed. trial and demographic data. In: Chadwick $\mathrm{D}$, ed. Proceedings of the fourth international symposium on sodium
valproate and epilepsy. London: Royal Society of valproate and epilepsy. London:

9 Kay R. Treatment effects in competing-risk analysis in prostate cancer. Biometrics 1986;42:203-11.

10 Davidson, DLW. The adult EPITEG trial: a comparative multicentre clinical trial of sodium valproate and carbamazepine in adult onset epilepsy. Part 2: Adverse effects In: Chadwick D, ed. Proceedings of the fourth international symposium on sodium valproate and epilepsy. London Royal Society of Medicine Services, 1989:114-24.

11 Chadwick D. Diagnosis of epilepsy. Lancet 1990:36: 291-6.

12 Heller AJ, Chesterman P, Elwes RDC, Reynolds EH, Crawford P, Chadwick D, Johnson AL. Phenobarbitone, phenytoin, carbamazepine or sodium valproate for newly diagnosed adult epilepsy: a randomised, comparative monotherapy trial. F Neurol Neurosurg Psychiatry 1994;

13 de Silva M, McArdle B, McGowan M, Neville BGR, Johnson AL, Reynolds EH. A prospective, randomised comparative monotherapy clinical trial in childhood epilepsy. In: Chadwick D, ed. Proceedings of the fourth international symposium on sodium valproate and epilepsy. London: Royal Society of Medicine Services, 1989: 81-6.

14 Verity CM, Hosking F, Easter DJ. A multicentre comparative trial of sodium valproate and carbamazepine in paediatric epilepsy. Dev Med Child Neurol 1994 (in press).

15 Mattson RH, Cramer JA, Collins JF and the Department of Veterans Affairs Epilepsy Cooperative Study No 264 Group. A comparison of valproate with carbamazepine for the treatment of complex partial seizures and secondarily generalized tonic-clonic seizures in adults. $N$ Engl f Med 1992;327:765-71.

15 Brodie MJ. Established anticonvulsants and treatment of refractory epilepsy. Lancet 1990;336:350-5. 